\title{
The influence of secondary wrong wiring of voltage transformer on measurement error
}

\author{
Suya $\mathrm{Li}^{1}$, Zhao Sun ${ }^{1}$, Lin $\mathrm{Li}^{1}$, Meng $\mathrm{Cao}^{1}$, Jufang Wei ${ }^{1}$ and Cong Zhao ${ }^{1}$ \\ ${ }^{1}$ State Grid Tianjin Electric Power Research Institute, Tianjin, China
}

\begin{abstract}
In this paper, the influence of mutual opposition error between the standard secondary line at the end of the test bench and the tested secondary line in the error test of voltage transformer is analyzed, and the corresponding solutions are proposed for the wrong wiring.
\end{abstract}

\section{Introduction}

Voltage transformer is the key equipment of power system, The voltage transformer handover test is an effective detection method in the equipment handover process, and the error measurement is an important verification item in the voltage transformer handover test.[1] During a routine inspection of $10 \mathrm{kV}$ voltage transformers, technicians found that the errors obtained in this batch of voltage transformer error tests did not match the voltage transformer error characteristics. In the article, we analyze the problems that occurred during the test, find the root cause of the error, conduct in-depth research, and give corresponding solutions.[2]

\section{Test phenomenon analysis}

The equipment used in this test includes: HES-1C test bench, regulator, booster, $10 \mathrm{kV}$ voltage transformer standard, load box. The test phenomenon is as follows: no alarm signal is generated during the test, the ratio difference in the fully loaded data is positive, showing an upward trend, angle difference is negative, showing a downward trend. In the light load data, the ratio difference and angle difference are both negative. According to the phenomenon of this test, the technicians look for the cause of the failure.Common problems during the error test include reverse polarity, secondary open circuit, wrong wiring, etc.The test bench HES-1C has a polarity check function. When the error test is normal wiring, it can check whether the polarity of the transformer is correct.[3]

If the polarity is abnormal, an error alarm will be generated when the voltage is raised with a voltage regulator. Since there is no alarm signal in this test, the problem of reverse polarity is first ruled out. [4]Because of the abnormal error in this test, the test bench, voltage regulator, booster, voltage transformer and load box are replaced and sent for inspection in turn, and no

E-mail: lisuya_work@163.com abnormality is found. Then the test wiring is carefully checked, and it is finally found that the source of the error is the standard secondary line T_0 of the voltage transformer at the test bench and the tested secondary line T_x, which causes an abnormal error. This article analyzes and discusses this kind of wrong wiring in depth.[5]

\section{Problem analysis}

When testing the voltage transformer in the laboratory, the technician compares the measured voltage transformer with the standard voltage transformer of the same rated transformation ratio.

The booster supplies the same primary voltage of the standard voltage transformer and the measured voltage transformer are supplied with the same primary voltage from the booster. The secondary voltage of the standard voltage transformer passes through the standard circuit of the transformer calibrator. The difference between the secondary voltage of the measured voltage transformer and passes through the differential loop of the transformer calibrator. Error data is read by transformer and calibrator.[6]

This test uses the measurement error of the low potential end of the comparison circuit, the voltage transformer verification circuit diagram is shown in Figure 1 below.In the picture is a voltage regulator, used to adjust the output voltage.is a booster, which cooperates with a voltage regulator to provide the measured voltage transformer plus the rated load, is a standard voltage transformer, and its voltage ratio is the same as the voltage transformer under test. HE is a transformer calibrator, used to test the ratio of the differential voltage to the phasor of the secondary voltage, that is, the error of the tested voltage transformer relative to the standard voltage transformer. When the voltage transformer standard secondary line and the tested secondary line are exchanged at the end of the error measuring device, the wiring diagram is shown in Figure 2. [7]We carry out two error measurements for 
correct wiring and wrong wiring of a batch of transformers. Table 1 is the measurement data of Dalian
Jin ye voltage transformer A, B, C.

Table. 1 10kV Error data of correct wiring and wrong wiring of voltage transformer

\begin{tabular}{|c|c|c|c|c|c|c|}
\hline $\begin{array}{c}\text { Serial } \\
\text { number }\end{array}$ & $\begin{array}{l}\text { Secondary } \\
\text { load }\end{array}$ & $\begin{array}{c}\text { Rated } \\
\text { voltage } \\
\text { percentage }\end{array}$ & $\begin{array}{l}\text { Correct } \\
\text { wiringe }\end{array}$ & $\begin{array}{l}\text { Correct } \\
\text { wiring } \delta\end{array}$ & $\begin{array}{c}\text { Wrong } \\
\text { wiringe }\end{array}$ & $\begin{array}{c}\text { Wrong } \\
\text { wiring } \delta\end{array}$ \\
\hline \multirow{4}{*}{002647} & \multirow{3}{*}{$30 \mathrm{VA}$} & $80 \%$ & -0.103 & +1.7 & +0.104 & -1.7 \\
\hline & & $100 \%$ & -0.108 & $\begin{array}{l}+1.7 \\
\end{array}$ & +0.105 & -1.7 \\
\hline & & $120 \%$ & -0.108 & +1.9 & +0.109 & -1.9 \\
\hline & $3.75 \mathrm{VA}$ & $100 \%$ & +0.160 & +0.5 & -0.159 & -0.5 \\
\hline \multirow{4}{*}{002573} & \multirow{3}{*}{$30 \mathrm{VA}$} & $80 \%$ & -0.104 & +1.9 & +0.109 & -2.0 \\
\hline & & $100 \%$ & -0.112 & +2.2 & +0.119 & -2.3 \\
\hline & & $120 \%$ & -0.121 & +2.5 & +0.123 & -2.5 \\
\hline & $3.75 \mathrm{VA}$ & $100 \%$ & +0.150 & +0.8 & -0.147 & -0.8 \\
\hline \multirow{4}{*}{002609} & \multirow{3}{*}{$30 \mathrm{VA}$} & $80 \%$ & -0.105 & +2.1 & +0.110 & -2.1 \\
\hline & & $100 \%$ & -0.108 & +2.2 & +0.111 & -2.2 \\
\hline & & $120 \%$ & -0.113 & +2.4 & +0.115 & -2.4 \\
\hline & $3.75 \mathrm{VA}$ & $100 \%$ & +0.159 & +0.6 & -0.155 & -0.6 \\
\hline
\end{tabular}

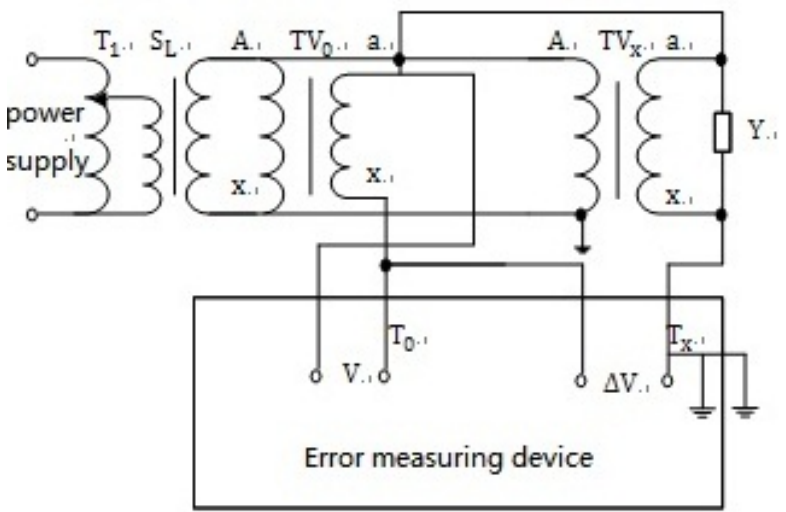

Figure 1 Correct wiring and compare lines (Low potential end measurement error)

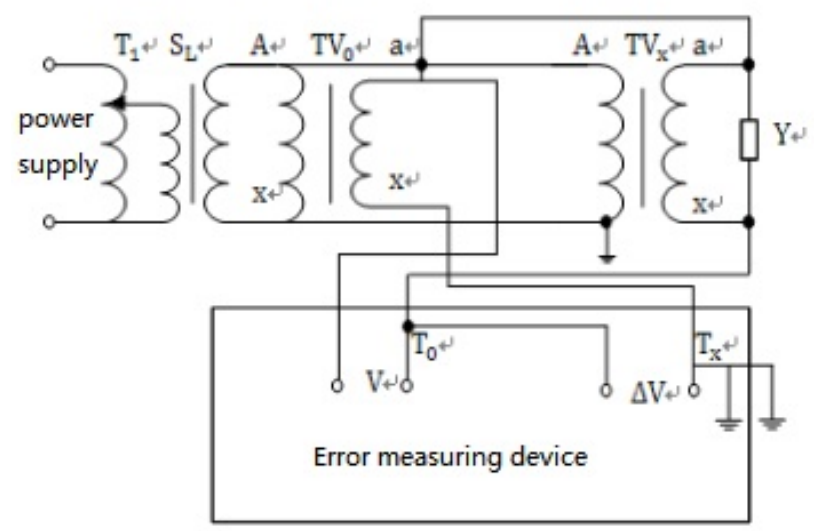

Figure 2 Wrong wiring comparison line (Low potential end measurement error)

Set the secondary voltage $\dot{U}_{20}$ of the standard voltage transformer as the working voltage: $\dot{U}=\dot{U}_{20}$

$$
\begin{aligned}
& \Delta \dot{\mathrm{U}}=\dot{\mathrm{U}}_{2 x}-\dot{\mathrm{U}}_{20} \\
& \Delta \dot{\mathrm{U}}=\Delta \mathrm{U}_{\mathrm{r}}+\mathrm{j} \Delta \mathrm{U}_{\mathrm{x}}
\end{aligned}
$$

So the ratio difference and angle difference of the tested voltage transformer are:

$$
\begin{aligned}
& \mathrm{f}_{\mathrm{u}}=\frac{\Delta \mathrm{U}_{\mathrm{r}}}{\mathrm{U}_{20}} \\
& \delta_{\mathrm{u}}=\frac{\Delta \mathrm{U}_{\mathrm{x}}}{\mathrm{U}_{20}}
\end{aligned}
$$

$\mathrm{K}_{\mathrm{u}}$-- The switching coefficient when measuring the error of the voltage transformer.

$\mathrm{K}_{\mathrm{x}}, \mathrm{K}_{\mathrm{y}}-\mathrm{T}$ The degree of the calibrator in-phase disk and quadrature disk.

When $\mathrm{T}_{0}$ and $\mathrm{T}_{\mathrm{x}}$ are reversed, the measured error is as follows:

$$
\begin{gathered}
\Delta \dot{\mathrm{U}}_{\mathrm{w}}=\dot{\mathrm{U}}_{20}-\dot{\mathrm{U}}_{2 \mathrm{x}}=-\Delta \dot{\mathrm{U}} \\
\Delta \dot{\mathrm{U}}_{\mathrm{w}}=\Delta \mathrm{U}_{\mathrm{rw}}+\mathrm{j} \Delta \mathrm{U}_{\mathrm{xw}}=-\Delta \mathrm{U}_{\mathrm{r}}+\mathrm{j}\left(-\Delta \mathrm{U}_{\mathrm{x}}\right) \\
\Delta \mathrm{U}_{\mathrm{rw}}=-\Delta \mathrm{U}_{\mathrm{r}} \\
\Delta \mathrm{U}_{\mathrm{xw}}=-\Delta \mathrm{U}_{\mathrm{x}} \\
\dot{\mathrm{U}}_{\mathrm{w}}=\dot{\mathrm{U}}_{2 \mathrm{x}}
\end{gathered}
$$

So the ratio difference and angle difference of the tested voltage transformer are:

$$
\begin{gathered}
\mathrm{f}_{\mathrm{uw}}=\frac{\Delta \mathrm{U}_{\mathrm{rw}}}{\mathrm{U}_{2 \mathrm{x}}}=\frac{-\Delta \mathrm{U}_{\mathrm{r}}}{\mathrm{U}_{2 \mathrm{x}}}=\frac{-\Delta \mathrm{U}_{\mathrm{r}}}{\mathrm{U}_{20}} \times \frac{\mathrm{U}_{20}}{\mathrm{U}_{2 \mathrm{x}}}=\frac{-\Delta \mathrm{U}_{\mathrm{r}}}{\mathrm{U}_{20}}\left(1+\frac{\mathrm{U}_{20}-\mathrm{U}_{2 \mathrm{x}}}{\mathrm{U}_{2 \mathrm{x}}}\right)= \\
-\frac{\Delta \mathrm{U}_{\mathrm{r}}}{\mathrm{U}_{20}}\left(1+\mathrm{K}_{\mathrm{p}}\right) \\
\delta_{\mathrm{uw}}=\frac{\Delta \mathrm{U}_{\mathrm{xw}}}{\mathrm{U}_{\mathrm{x}}} \times 3438=\frac{-\Delta \mathrm{U}_{\mathrm{x}}}{\mathrm{U}_{2 \mathrm{x}}}=\frac{-\Delta \mathrm{U}_{\mathrm{x}}}{\mathrm{U}_{20}} \times \frac{\mathrm{U}_{20}}{\mathrm{U}_{2 \mathrm{x}}}=\frac{-\Delta \mathrm{U}_{\mathrm{x}}}{\mathrm{U}_{20}}(1+ \\
\left.\frac{\mathrm{U}_{20}-\mathrm{U}_{2 \mathrm{x}}}{\mathrm{U}_{2 \mathrm{x}}}\right)=-\frac{\Delta \mathrm{U}_{\mathrm{x}}}{\mathrm{U}_{20}}\left(1+\mathrm{K}_{\mathrm{p}}\right) \\
\mathrm{K}_{\mathrm{p}}=\frac{\mathrm{U}_{20}-\mathrm{U}_{2 \mathrm{x}}}{\mathrm{U}_{2 \mathrm{x}}}
\end{gathered}
$$

According to "Measurement Voltage Transformer" JJG314-2010 regulation, the standard device should be two higher accuracy levels than the tested voltage transformer, The actual error should not be greater than $1 / 5$ of the error limit of the tested voltage transformer. The daily verification of the voltage transformer level is 0.2 ,then the standard transformer standard is 0.05 . That is: $\left|K_{\mathrm{p}}\right| \leq \frac{0.05+0.2}{(1-0.05) \times(1+0.2)}=\frac{25}{114} \approx 0.22$. 


\section{The curve diagram of transformer A, B,} C full load ratio difference

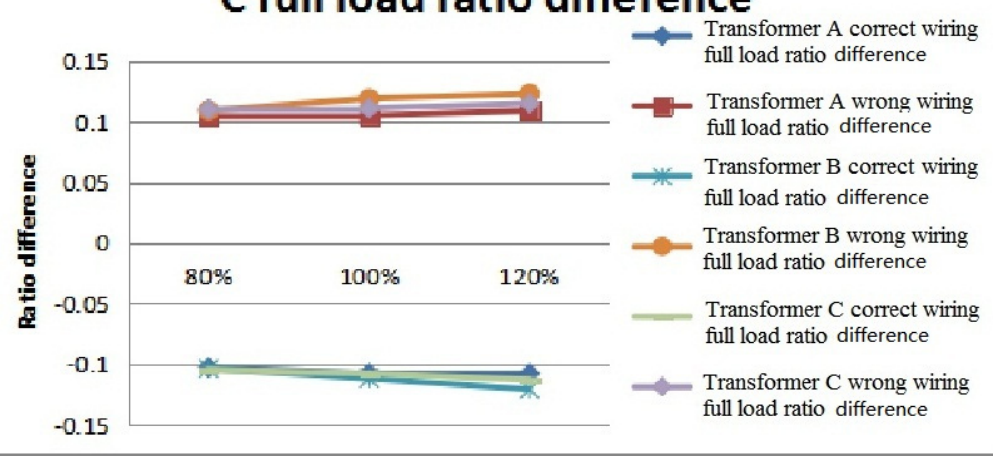

Figure 3 The curve diagram of transformer A, B, C full load ratio difference

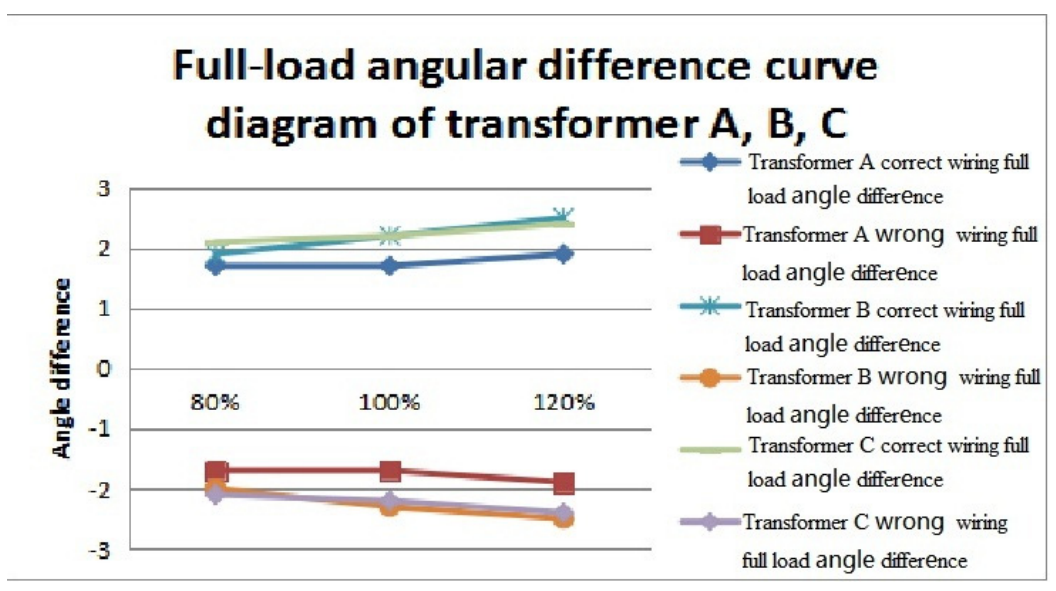

Figure 4 Full-load angular difference curve diagram of transformer A, B, C

Figure 3 and Figure 4 are the comparison diagrams of the ratio difference and the angle difference between the correct and incorrect wiring of the voltage transformer in Table 1.It can be clearly seen from Fig. 3 and Fig. 4 and formulas (9) and (10), after wrong wiring, the signs of the ratio and angle differences are opposite to those of the correct wiring, and the absolute value is not much different.[8] When the wiring is correct, the full load ratio difference is negative, and decreases with the increase of voltage,angle difference is positive and increases with the increase of voltage. The light load ratio difference is positive, and the angle difference is positive, When $\mathrm{T}_{0}$ and $\mathrm{T}_{\mathrm{x}}$ are reversed, the measured full load ratio difference is positive, and it increases with the increase of the positive voltage,the angle difference is negative and decreases with the increase of voltage,the light load ratio difference is negative, the angle difference is negative.[9]

\section{Solution}

Wrong wiring in the transformer error experiment, especially the wrong wiring at the end of the calibration station, often occurs during equipment replacement and equipment inspection, but this type of wrong wiring is slightly different from the wrong wiring of the tested terminal.The error of the voltage transformer tested by this type of wrong wiring will not have wrong polarity, not easy to appear out of tolerance, and easy to be ignored, which affects the fairness, accuracy and reliability of the verification result to a certain extent.In order to effectively solve this problem, you can mark the wiring at the end of the error experiment test bench, or use different color lines to distinguish, to prevent wrong wiring during the equipment inspection or replacement process, which affects the detection accuracy.[10]

\section{Conclusion}

The above analysis of the abnormal error is caused by the wrong wiring of the voltage transformer in a laboratory, and corresponding solutions are proposed. In daily calibration work, the verification principle and verification circuit of general transformers should be proficient. For commonly used transformer testers, standard transformers, load boxes, etc., they should be verified regularly to ensure that the verification results are accurate.

\section{Acknowledgments}

Great thanks to the funding of State Grid Corporation of China Standard Compilation Project (KJ20-2-09): Guide for condition evaluation of inductive voltage transformer. 


\section{References}

1. Teng Wentao,Xiang Zutao,Zheng Bin,Song Xiuyou,Zhang Yuanyuan,Li Kuan. Study on Factors Affecting Residual Magnetism of Phase Selection of Extreme High Voltage Transformer and Its Calculation Method2019:55-61.

2. Dingzhen Li,Jianchang Guo,Jing Fan. Research on the Combination of Electromagnetic Voltage Transformer and Straight-through Current Transformer[C]. Institute of Management Science and Industrial Engineering.Proceedings of 2019 2nd International Conference on Intelligent Systems Research and Mechatronics Engineering(ISRME 2019).Institute of Management Science and Industrial Engineering2019:206-211

3. Effect of Series Capacitor Compensation in Power Transmission System Composed of Power Line and Shield Wires in Parallel[J]. Katsuo Isaka,Atsuo Chiba,Yukio Onogi. 1981(8)

4. Linear and Nonlinear Dielectric Ceramics for High-Power Energy Storage Capacitor Applications[J]. Journal of the Korean Ceramic Society.2019(1)
5. Yang Binwen, Li Wensheng 2010 Causes of PT ferroresonance and countermeasures Electric Power Auto. Equip.30 134-36

6. Implementation of Power Optimization Technique for UAVs[J]. Pirati Gangadhara Sai,Chapala Sandhya Rani,Usha Rani Nelakuditi. Materials Today: Proceedings. 2018(1)

7. Research on Residual Magnetic Resonance Detection Method Based on Time-Current Curve. Niu Shuaijie,Zhao Lihua,Chen Ling,et al. Electric Measuring\&Instrumentation. 2017

8. Research on Identification of Transformer Magnetizing Inductance Using Hysteresis Loop. He Yuan,Li Xin,Luo Jian. Power System Protection and Control.2013

9. Engineering-Power Systems; Findings on Power Systems Reported by Investigators at Indian Institute of Technology (A Model-Free Approach for Emergency Damping Control Using Wide Area Measurements)[J].2015

10. Engineering - Power Systems; Researchers at Carnegie Mellon University Report New Data on Power Systems (Equivalent Circuit Formulation for Solving Ac Optimal Power Flow)[J]. 2016 